\title{
Rheumatoid Arthritis with Neurological Involvement Manifested as Hemispheric Masses
}

\author{
Pei-Lin Tsai $^{a}$ Jiann-Horng Yeh ${ }^{a, c}$ Wei-Hung Chen ${ }^{a, d}$ Chin-Cheng Lee ${ }^{b}$ \\ ${ }^{a}$ Department of Neurology and ${ }^{b}$ Department of Pathology and Laboratory Medicine, \\ Shin Kong Wu Ho-Su Memorial Hospital, ' College of Medicine, Fu-Jen Catholic University, and \\ ${ }^{\mathrm{d} C}$ College of Medicine, Taipei Medical University, Taipei, Taiwan, ROC
}

Dear Sir,

Central nervous system (CNS) involvement in patients with rheumatoid arthritis (RA) is rare. The pathological findings include rheumatoid nodule, pachy- or leptomeningitis, and vasculitis of necrotizing or plexiform type [1-3]. We report a patient with the unusual presentation of multiple masses in the cerebral hemispheres which were found to be associated with lymphocytic infiltration of some cerebral vessel walls. The masses regressed after steroid treatment.

\section{Case Report}

A 58-year-old right-handed male farmer had a 3-year history of RA manifested as intermittent painful swelling of the ankles and interphalangeal joints of both feet (fig. 1a), and an elevated rheumatoid factor titer of $197 \mathrm{IU} / \mathrm{ml}$ (normal value $<30$ $\mathrm{IU} / \mathrm{ml})$. He took only analgesics for symptom relief. He did not take any diseasemodifying antirheumatic drugs or steroids. One month prior to admission, he started to experience painful swelling in his right wrist, which was followed by slurred speech and progressive weakness in his right limbs. At admission, he developed a low-grade fever and polyarthritis in both of his knees, ankles, shoulders, and right wrist. Neurological examination revealed that he had anomia, visual agnosia, mild right central facial palsy, right hemiparesis (Medical Research Council, MRC, grade 4/5), and right hemihypesthesia of all sensory modalities. Laboratory findings were unremarkable except for an elevated level of rheumatoid factor (238 IU/ml). A brain MRI scan disclosed 3 mass lesions in the bilateral temporal and the left periventricular regions. The lesions were hypointense in $\mathrm{T}_{1}$-weighted imaging, hyperintense in $\mathrm{T}_{2}$-weighted imaging (fig. 1b, c), and were not enhanced by gadolinium. The patient underwent a stereotactic biopsy of his periventricular lesion. The pathology revealed perivascular lymphocytic infiltration and reactive gliosis (fig. 1d, e), which were compatible with vasculitis. We initiated pulse therapy of methylprednisolone (1 g/day); however, the patient developed steroid psychosis 2 days later. We therefore discontinued the pulse therapy and administered oral prednisolone $60 \mathrm{mg} /$ day instead. After $1 \mathrm{month}$ of therapy, follow-up MRI disclosed regression of the 3 brain lesions (fig. 1f), and his symptoms improved with residual minimal right hemiparesis (MRC 4+/5). The patient's clinical features remained stable during the subsequent 3 years of follow-up.

\section{Discussion}

Direct CNS involvement in patients with RA is rare. The well-known condition of rheumatoid vasculitis typically involves the skin and peripheral nerves, rarely does it affect the CNS. In addition, although nodular formation is a frequent and characteristic extra-articular feature of RA, nodular formation within the CNS is uncommon. Bathon et al. [1] reported on 19 patients with seropositive long-standing RA who developed inflammatory CNS lesions. In these patients, rheumatoid nodules were the most common pathological finding (68\%), followed by lepto- or pachymeningeal inflammation (63\%) and vasculitis (37\%). These 3 pathological changes were present with similar frequencies in symptomatic patients, but CNS nodules alone were often found in asymptomatic patients. CNS disease occurs in a significant number of patients without active synovitis and extracranial vasculitis and nodules. CNS vasculitis in patients with RA may manifest as necrotizing vasculitis [4] or arterial plexiform change with amyloidosis [5]. These findings, however, were not observed in our patient.

Recently, Tajima et al. [6] reported a case of RA with multiple CNS lesions that resolved after steroid treatment. Regard-

\section{KARGER}

Fax +41 613061234

E-Mail karger@karger.ch

www.karger.com (c) 2008 S. Karger AG, Basel

0014-3022/08/0594-0190\$24.50/0

Accessible online at:

www.karger.com/ene
Wei-Hung Chen, MD

Department of Neurology

Shin Kong Wu Ho-Su Memorial Hospital

95 Wen-Chang Road, Shih-Lin District, Taipei, Taiwan (ROC)

Tel +886 22833 2211, ext. 2595, Fax +886 2834 4906, E-Mail M000735@ms.skh.org.tw 

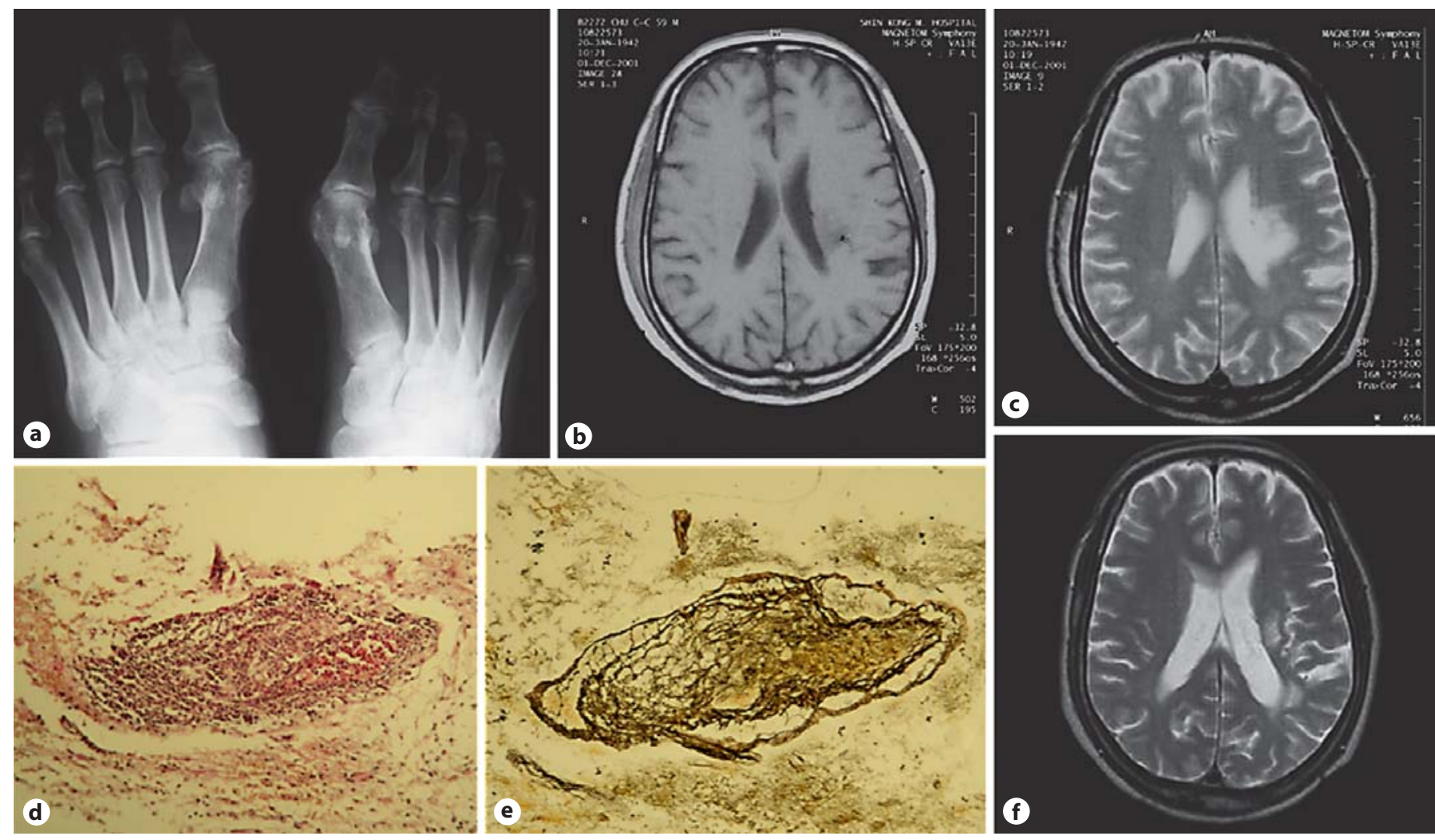

Fig. 1. a The radiograph shows eroded articular cartilages of the proximal and distal interphalangeal joints of both feet. b An MRI $\mathrm{T}_{1}$-weighted axial image shows 2 of the 3 lesions in this patient: one in the left periventricular region and the other in the left angular gyrus. Both lesions are hypointense. $\mathbf{c}$ In a $\mathrm{T}_{2}$-weighted image, the

lesions are hyperintense. $\mathbf{d}$ The pathology shows perivascular lymphocytic infiltration around a vessel. HE. $\times 100$. e Reticulin staining of the lesion shows increased reticular fibers in a thickened vessel wall. Reticulin stain. $\times 100 . \mathbf{f}$ In a follow-up $\mathrm{T}_{2}$-weighted image, the lesion in the periventricular region has regressed.

ing the MRI findings ( $\mathrm{T}_{1}$ hypointense, $\mathrm{T}_{2}$ hyperintense, diffusion-weighted image isointense and lack of gadolinium enhancement), they suggested that the lesions were attributable to vasogenic edema. However, the causes of vasogenic edema were not discussed. In our case, the finding of perivascular lymphocytic infiltration may be responsible for breakdown of the blood-brain barrier and subsequent vasogenic edema, which in turn produces neurological symptoms. Steroid use is effective for reducing vasogenic edema and suppressing the inflammatory process. We consider that perivascular lymphocytic infiltration is a form of vasculitis caused by RA, but is not as severe as in those cases reported previously.
In conclusion, we report a rare case of RA with multiple lesions in the brain involving both the gray and white matters of both hemispheres. The lesions were considered as vasogenic edema caused by a mild form of vasculitis that has not been reported previously.

\section{References}

1 Bathon JM, Moreland LW, DiBartolomeo AG: Inflammatory central nervous system involvement in rheumatoid arthritis. Semin Arthritis Rheum 1989;18:258-266.

2 Cellerini M, Gabbrielli S, Maddali Bongi S, Cammelli D: MRI of cerebral rheumatoid pachymeningitis: report of two cases with follow-up. Neuroradiology 2001;43:147150.
3 Kamio N, Kuramochi S, Wang RJ, Hirose S, Hosoda Y: Rheumatoid arthritis complicated by pachy- and leptomeningeal rheumatoid nodule-like granulomas and systemic vasculitis. Pathol Int 1996;46:526-530.

4 Watson P, Fekete J, Deck J: Central nervous system vasculitis in rheumatoid arthritis. Can J Neurol Sci 1977;4:269-272.

5 Matsuki Y, Suzuki K, Tanaka N, Hirose T, Hosoai K, Kawakami M, Ishizuka T, Kawaguchi Y, Hidaka T, Nakamura H: Amyloidosis secondary to rheumatoid arthritis associated with plexiform change in bilateral temporal lobes. Intern Med 1994;33:764767.

6 Tajima Y, Kishimoto R, Sudoh K, Matsumoto A: Multiple central nervous system lesions associated with rheumatoid arthritis. Arch Neurol 2004;61:1794-1795. 